\title{
A Two-Stage Compression Method for the Fault Detection of Roller Bearings
}

\author{
Huaqing Wang, Yanliang Ke, Ganggang Luo, Lingyang Li, and Gang Tang \\ School of Mechanical and Electrical Engineering, Beijing University of Chemical Technology, Beijing 100029, China \\ Correspondence should be addressed to Gang Tang; tanggang@mail.buct.edu.cn
}

Received 22 January 2016; Revised 10 April 2016; Accepted 8 May 2016

Academic Editor: Daniel M. Sotelo

Copyright ( 2016 Huaqing Wang et al. This is an open access article distributed under the Creative Commons Attribution License, which permits unrestricted use, distribution, and reproduction in any medium, provided the original work is properly cited.

\begin{abstract}
Data measurement of roller bearings condition monitoring is carried out based on the Shannon sampling theorem, resulting in massive amounts of redundant information, which will lead to a big-data problem increasing the difficulty of roller bearing fault diagnosis. To overcome the aforementioned shortcoming, a two-stage compressed fault detection strategy is proposed in this study. First, a sliding window is utilized to divide the original signals into several segments and a selected symptom parameter is employed to represent each segment, through which a symptom parameter wave can be obtained and the raw vibration signals are compressed to a certain level with the faulty information remaining. Second, a fault detection scheme based on the compressed sensing is applied to extract the fault features, which can compress the symptom parameter wave thoroughly with a random matrix called the measurement matrix. The experimental results validate the effectiveness of the proposed method and the comparison of the three selected symptom parameters is also presented in this paper.
\end{abstract}

\section{Introduction}

Rotating machinery is widely applied in industrial fields, such as petrochemical industry, metallurgy industry, and power industry $[1,2]$. Roller bearing, whose failure might result in the breakdown of the whole mechanical system, is considered as an integral component of rotating machinery $[3,4]$. Therefore, it is essential to monitor the operating condition of the roller bearings, aiming at preventing the occurrence of the accidents.

Since much fault information is carried by the vibration signals, vibration-based diagnostic techniques have become the most commonly used and effective method for the fault diagnosis of roller bearings [5-7]. It is well known that the vibration-based fault diagnosis of roller bearings can be broadly classified into three categories, namely, time-domain analysis, frequency-domain analysis, and time-frequency analysis [8-10].

In the case of time-domain analysis, the characteristic statistical factors in time domain, including mean, peak, root mean square, skewness, can be viewed as descriptors to assess the performance of roller bearings [11-13]. Wang et al. [14] proposed a comprehensive analysis based on time-domain and frequency-domain statistical factors in order to evaluate the performance degradation degree of roller bearings. Niu et al. [15] presented some new statistical moments for the early detection of bearing failure. Heng and Nor [16] investigated a statistical method to detect the presence of defects in a roller bearing. Although the symptom parameters are easily performed to evaluate the condition of roller bearings, the successful applications are limited owing to the weak antiinterference performance of these parameters.

Compared to time-domain analysis, frequency-domain analysis has an advantage in highlighting the certain frequency components of interest by transforming the timedomain wave into frequency spectrum. The traditional method in frequency-domain is fast Fourier transform (FFT). Due to the modulation phenomenon of faulty vibration signals, envelope demodulation should be carried out before performing FFT, which is called envelope analysis. Guo et al. [17] applied envelope analysis with independent component analysis, which can extract the impulse component 
corresponding to the roller bearing faults and reduce the dimension of vibration sources. Wang et al. [18] developed a fault detection enhancement method based on the peak transform and envelope analysis. Cases proved that the envelope analysis can diagnose the faults of a roller bearing successfully when it is in combination with other approaches [17-19]. However, the frequency analysis will lose its effect when the vibration signals are embedded in strong noise. Thus, the time-frequency-domain analysis was developed. Many methods that belong to time-frequency analysis, such as empirical mode decomposition (EMD) [20] and wavelet transform [21], are employed to assess the performance of roller bearings. Ma et al. [22] combined local mean decomposition and time-frequency analysis, which can improve the reliability of the fault diagnosis. Li et al. [23] proposed a novel method for fault diagnosis of roller bearings based on CEEMD. Ahn et al. [24] applied wavelet analysis to eliminate noise. Though time-frequency analysis is effective in processing the nonstationary signals induced by faulty bearings, it is usually complicated and involves large computation, which is contrary to the real-time detection.

The cited literatures demonstrated that the fault diagnosis of roller bearings is developing increasingly. However, the aforementioned bearing fault diagnosis method is achieved by sampling the vibration signals under the Shannon sampling theorem. With the constraint of Shannon sampling theorem, a large amount of redundant vibration signals will be measured, increasing the burden of roller bearings' fault diagnosis. There is no doubt that the increasing amount of data will result in high accuracy of fault diagnosis with the efficiency decreasing. Thus, it is a really tough work to balance the accuracy and efficiency of fault diagnosis.

A newly developed theory named compressed sensing (CS) [25] brought a new insight to deal with the bigdata problem, which puzzled the researchers in various fields. The core idea of the CS theory is to reconstruct the original signals from a small number of samples far below the Shannon sampling rate using sparse representation and a well-designed measurement matrix. The CS theory has been applied to numerous fields, such as image processing, medical field, and remote sensing. Khwaja and Ma [26] described two possible applications of the CS theory in synthetic aperture radar image compression. Zhu et al. [27] developed an adaptive sampling mechanism on the blockbased CS, which focused on how to improve the sampling efficiency for CS-based image compression. Kim and Vu [28] applied the CS theory to magnetic resonance imaging, which can be viewed as a breakthrough technology in medical diagnosis. Ghahremani and Ghassemian [29] combined the ripplet transform and the CS theory to remote sensing image fusion. All of the aforementioned studies demonstrated the possibility of applying the CS theory to the field of fault diagnosis. However, the applications of the CS theory in fault diagnosis are relatively limited. Although Zhu et al. [30] summarized the applications in the mechanical fields, no practical applications were reported. Chen et al. [31] presented a novel adaptive dictionary based on the CS theory to extract the impulse generated by the faulty bearings. Tang et al. [32] developed a representation classification strategy for rotating machinery faults based on the CS theory. Wang et al. [33] proposed a novel decomposition for reconstruction from the limited observations polluted by noise based on the CS theory via the sparse time-frequency representation. However, the aforementioned studies were primarily focused on either the sparse representation of the vibration signals or the reconstruction of the original signals, and the amount of samples in these cases still needed to be compressed.

It is significant to ensure that the few observations contain adequate faulty information, which is an essential condition to guarantee the successful applications of the compressed sensing theory. Thus, a fault features' reservation method called the symptom parameter wave is developed to obtain sufficient faulty information. Combined with the compressed sensing theory, a two-stage compression method is described in this work to further decrease the amount of samples for fault diagnosis of roller bearings without losing significant information, through which the fault features of roller bearings can be detected timely. Compared to the work of sparse representation and reconstruction, the samples for the fault detection using the two-stage compression method are far less. First, the large amount of vibration signals is divided into several segments by a sliding window with a given size. Then, a time-domain symptom parameter is used to represent each data segment, through which a symptom parameter wave can be obtained and the original signals can be reduced to a certain level. With the symptom parameter wave, the dimension of the analyzed signals can be shrunk and it outperforms the traditional usage of these characteristic factors by representing the whole signals with single value in the presence of noise. Second, a well-designed measurement matrix is applied to compress the symptom parameter wave. Third, a fault detection method based on the CS theory is employed to extract the fault features with limited samples. Assisted by the matching pursuit, the fault features can be detected from a small number of samples, which are far below the Shannon sampling rate. Furthermore, the detection method in the current work does not need to reconstruct the original signals completely. When the components related to the fault features are detected, the reconstruction process can be finished, which means the fault diagnosis can be completed during the reconstruction procedure.

The rest of this paper is organized as follows. Section 2 introduces the basic concept of the two-stage compression strategy, followed by the compressed fault detection strategy in Section 3. The application cases are presented in Section 4 . Section 5 describes the comparing results between the selected symptom parameters. Conclusions are drawn in Section 6.

\section{Basic Concept of the Two-Stage Compression Strategy}

2.1. First Stage of Compression by the Symptom Parameter Wave. As is known to all, the fault diagnosis of roller bearings based on the time-domain symptom parameters is the simplest method in time-domain analysis. The operating status of roller bearings can be identified according to the change of the time-domain symptom parameters. Generally 
speaking, the symptom parameters can be classified into two categories: dimensional symptom parameters and nondimensional symptom parameters. The former, such as the peak value, the peak-to-peak value, and the root mean square value, reflect the magnitude change of a signal. The latter, such as kurtosis, crest factor, and shape factor, express the shape change of a signal.

Various symptom parameters have been utilized for fault diagnosis of roller bearings. Some of them can be calculated according to the following equations:

$$
\begin{aligned}
X_{\text {Peak }} & =\max \left\{x_{1}, x_{2}, \ldots, x_{N}\right\}, \\
\bar{X} & =\frac{1}{N} \sum_{i=1}^{N}\left|x_{i}\right|, \\
X_{\mathrm{RMS}} & =\sqrt{\frac{1}{N} \sum_{i=1}^{N} x_{i}^{2},} \\
X_{\mathrm{ptp}} & =x_{\max }-x_{\min }, \\
\mathrm{SF} & =\frac{X_{\mathrm{RMS}}}{\bar{X}}, \\
K & =\frac{(1 / N) \sum_{i=1}^{N} x_{i}^{4}}{X_{\mathrm{RMS}}^{4}},
\end{aligned}
$$

where $X_{\text {Peak }}$ is the peak value of a signal, $\bar{X}$ represents the average of absolute value of a signal, $X_{\mathrm{RMS}}$ denotes the root mean square of a signal, $X_{\mathrm{ptp}}$ expresses the peak-to-peak value of a signal, $x_{\max }$ is the max value of a signal, $x_{\min }$ is the minimum value of a signal, $\mathrm{SF}$ describes the shape factor of a signal, and $K$ indicates the kurtosis of a signal.

In the traditional sense, the operating status of roller bearing can be identified by representing the whole signals with a characteristic value. This fault diagnosis method mainly depends on the difference of the characteristic values between the normal state and faulty status. However, successful cases are limited due to the instability and insensitivity of these parameters when the target vibration signals are submerged by the noise, which means the traditional usage of these symptom parameters has a weak anti-interference ability. To strengthen the ability of antinoise, a concept of symptom parameter wave is proposed in the present study. Three time-domain symptom parameters are selected to represent the signals depending on the characteristics of the faulty vibration signals. The selected symptom parameters are $X_{\mathrm{ptp}}$, $\mathrm{SF}$, and $K$, which are more sensitive to the failures than other characteristic factors. A symptom parameter wave can be achieved through a sliding window in order to compress the raw signals and preserving the fault features of the faulty vibration signals. The flow diagram of symptom parameter wave is shown in Figure 1. The raw vibration signals are divided into several segments by a sliding window and a selected characteristic parameter is used to represent each segment, through which a symptom parameter wave can be obtained. The acquisition of the symptom parameter wave can reduce the original signals to a certain level with the fault

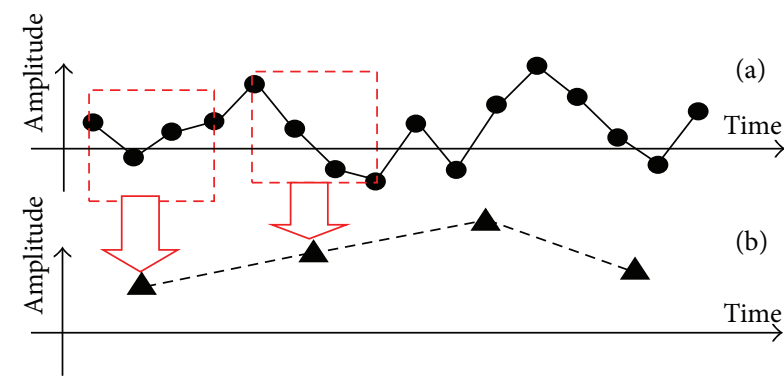

FIGURE 1: Flow diagram of obtaining the symptom parameter wave. (a) The original signals and (b) the symptom parameter wave.

features remaining, which is the first stage of compression in this work.

2.2. Second Stage of Compression by the Compressed Sensing Theory [34-36]. The traditional sampling scheme based on the Shannon sampling theorem results in the big data, which contains much redundant information. It is no doubt that large amount of signals increases the cost of data storage and makes signal processing more difficult. The proposal of the CS theory brings a new insight for data acquisition and signal processing by reconstructing the original signal from downsampled signals.

Provided $\mathrm{SP}(t)$ is a $N \times 1$ symptom parameter wave, which can be further compressed by a measurement matrix:

$$
y=\Phi S P(t),
$$

where $\Phi$ is a $M \times N(M \ll N)$ measurement matrix.

The $N \times 1$ time-domain signal can be compressed to a $M \times 1$ signal using a measurement matrix according to (2).

The $\mathrm{SP}(t)$ can be represented by a group of $N \times 1$ basis:

$$
\mathrm{SP}(t)=\psi \theta .
$$

So

$$
y=\Phi \psi \theta=A \theta,
$$

where $\psi=\left\{\varphi_{1}, \varphi_{2}, \ldots, \varphi_{N}\right\} \in N \times N$ is the basis vector, through which the original signals can be represented sparsely. $\theta$ denotes the representation coefficients. The signals can be said to be $K$-sparse, when $\theta$ contains only $K$ nonzero coefficients $(K \ll N) . A=\Phi \psi$ is called the sensing matrix.

It is impossible to solve the model in (3), which is an ill-posed equation. The solution of the CS model can be expressed as

$$
\begin{array}{ll}
\min & \|\theta\|_{0} \\
\text { s.t. } & y=A \theta .
\end{array}
$$

The solution becomes possible owing to the sparsity of $\theta$, which can reduce the unknowns. It is more likely to reconstruct the signals from the limited samples without meeting the requirement of Shannon sampling theorem, when $A$ satisfies the restricted isometry property (RIP). 


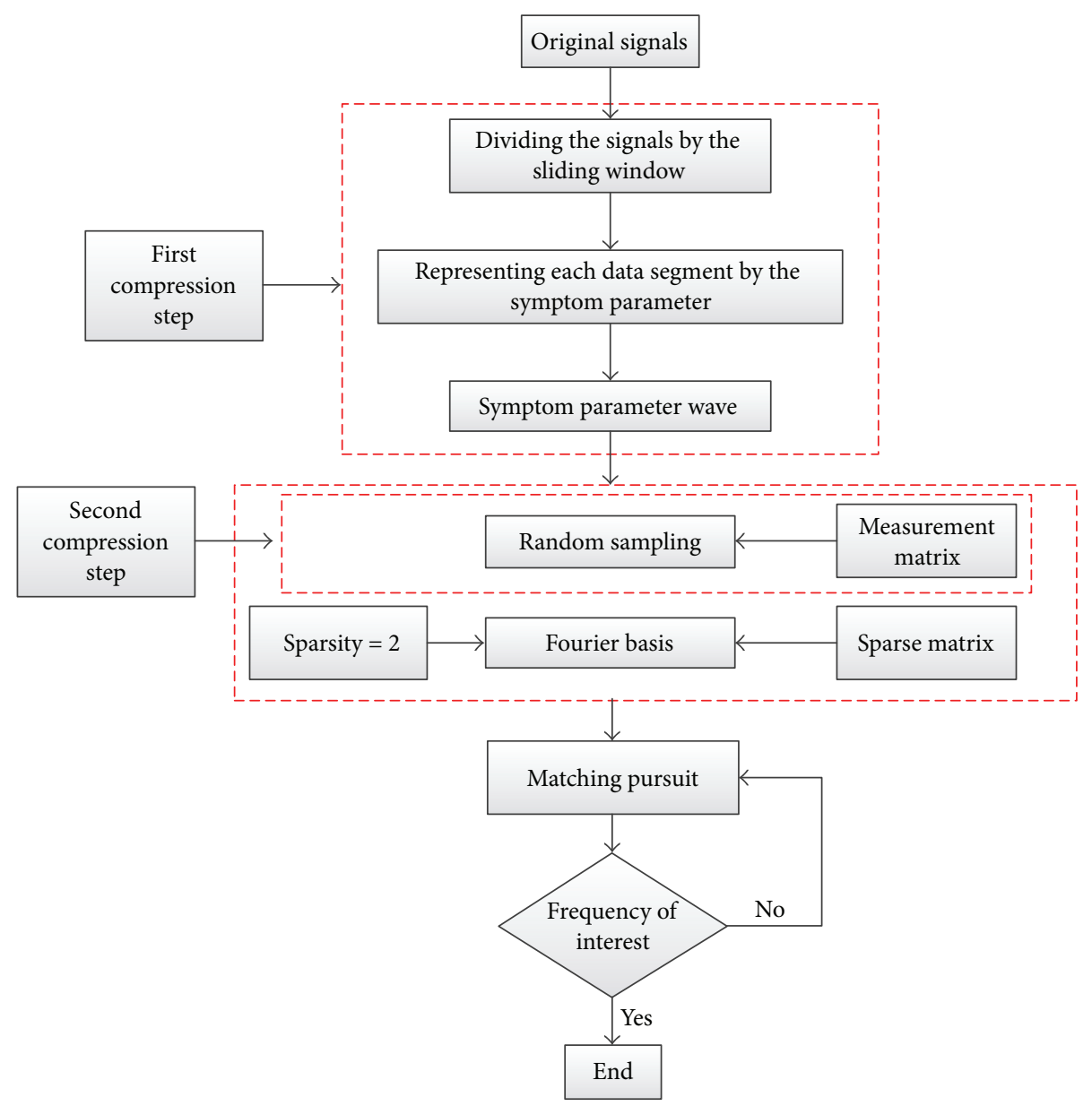

FIGURE 2: Flow diagram of the compressed fault detection strategy.

\section{The Compressed Fault Detection Strategy}

A force impulse is generated when operating a roller bearing with a local defect and the resonance of the bearing system occurs. In addition, each time the ball passes through the location with a fault, a periodic impulse will be produced, namely, the fault characteristic frequency, which is an indicator of the existence of a fault. Due to the phenomenon of resonance, the fault characteristic frequency will be modulated. Thus, the faulty vibration signals need to be demodulated in order to obtain the fault characteristic frequency.

An obstacle should be overcome in case of the application of the CS theory to fault diagnosis of roller bearings. In fact, the faulty vibration signals, which contain many periodic impulses, are not significantly sparse neither in time domain nor in Fourier domain. In this case, the reconstruction error will be high, which means that it makes no sense to reconstruct the signals. Thus, we choose to detect the harmonic signals induced by the faulty roller bearings. In addition, it is well known that the sparsity of the harmonic signal in Fourier domain is two. Furthermore, the advantage of the fault detection over the reconstruction is that if the frequency of the detected signal is of interest, the process can be completed, which can also be called the incomplete reconstruction.
The flowchart of the compressed fault detection approach in this work is presented in Figure 2. The amount of the original signals can be reduced by the time-domain symptom parameter wave through a size-fixed sliding window. As mentioned above, a variety of impulses exist in the faulty vibration signals and the peak-to-peak value, kurtosis, and shape factor are more suitable to represent the fault characteristics. Thus, the peak-to-peak value, kurtosis, and shape factor are chosen to obtain the symptom parameter wave. Then, the symptom parameter wave can be acquired and the dimension of the original signals is reduced to a certain level with the faulty information remaining. Additionally, the vibration signals of faulty bearing can be demodulated by the symptom parameter wave. Thus, it is unnecessary to adopt the envelope demodulation to process the analyzed signals. The measurement matrix is utilized as a compressor to further shrink the amount of samples. At last, fault detection based on the CS theory is employed to extract the fault features with the help of matching pursuit. If the detected harmonic signal is almost equal to the theoretical fault characteristic frequency, the conclusion that there is a fault in this roller bearing can be made. Otherwise, the detection process should be continued until the frequencies of interest are extracted. Here, the basis group is the Fourier basis for the sparsity of harmonic signal 


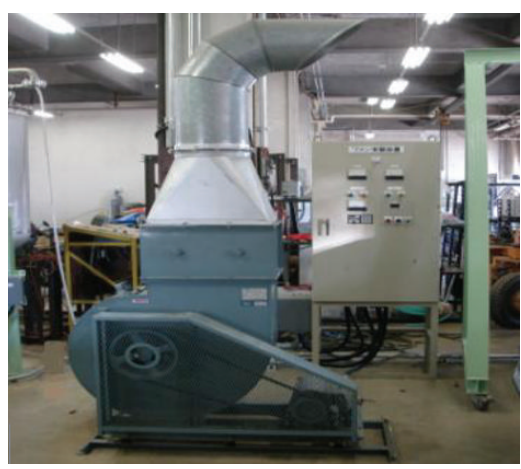

(a)

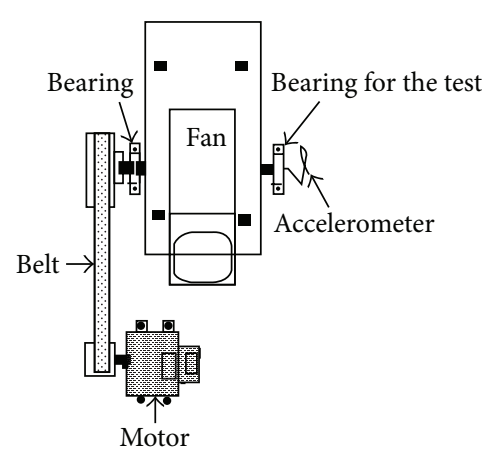

(b)

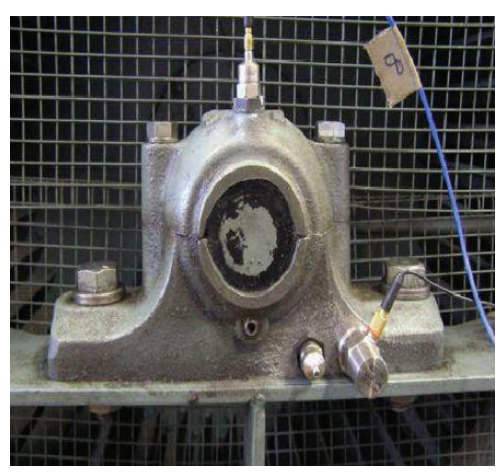

(c)

FIGURE 3: (a) Fault test rig, (b) flow diagram of the fault test rig, and (c) location of the sensors.

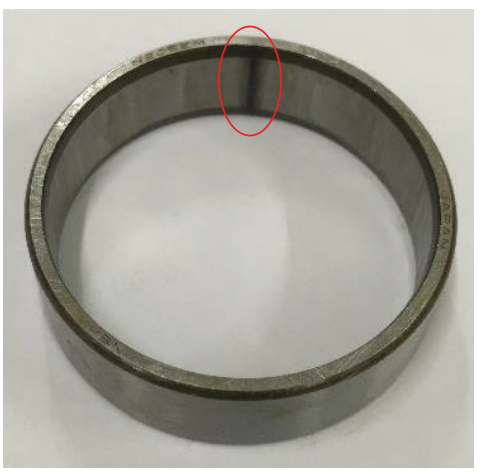

(a)

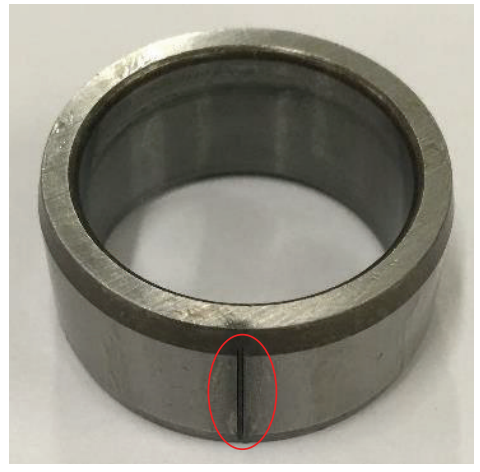

(b)

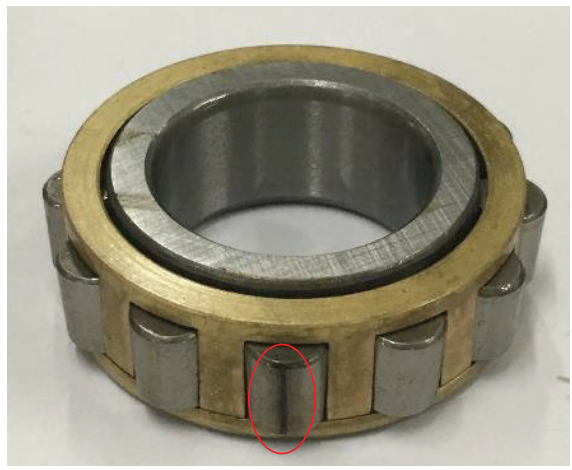

(c)

FIGURE 4: (a) Outer-race fault, (b) inner-race fault, and (c) rolling-element fault.

in Fourier domain is 2. Various matrices can be considered as measurement matrix, such as Gaussian random matrix, Bernoulli matrix, and Toeplitz matrix. Since the Gaussian random matrix can meet the requirement of RIP with the Fourier basis, it is selected as the measurement matrix in this study.

\section{Application Cases}

4.1. Test Rig. The vibration signals of roller bearing faults are measured through an accelerometer, and the faulty bearings are fixed in the fan system, as shown in Figure 3(a), which mainly consists of a motor, a belt, a fan, and a couple of bearings. The motor is a three phase induction motor, whose type is SF-JR with a rated power of $2.2 \mathrm{Kw}$. The fan, which has 48 blades, operates at a speed of $800 \mathrm{rpm}$. The flow diagram of the fan system is presented in Figure 3(b). The sensors are installed on the bearing housing, as shown in Figure 3(c), where the collected vibration signals might be valid and easily measured. The faulty bearings are shown in Figure 4, concluding outer-race fault, inner-race fault, and rolling-element fault, which are created through wire-electrode cutting. In all experiments, the sampling frequency is $100 \mathrm{KHz}$ and the roller bearings are operated at a speed of $800 \mathrm{rpm}$. The fault characteristic frequencies can be computed according to (6)(8) $[23,37]$ and the results are shown in Table 1.
TABLE 1: Fault characteristic frequency.

\begin{tabular}{lccc}
\hline Fault location & Outer race & Inner race & Ball element \\
\hline $\begin{array}{l}\text { Fault characteristic } \\
\text { frequency }(\mathrm{Hz})\end{array}$ & 72.5 & 103.4 & 79.6 \\
\hline
\end{tabular}

The fault characteristic frequency of outer race is

$$
f_{o}=\frac{Z}{2}\left(1-\frac{d}{D} \cos \alpha\right) f_{r}
$$

The fault characteristic frequency of inner race is

$$
f_{i}=\frac{Z}{2}\left(1+\frac{d}{D} \cos \alpha\right) f_{r}
$$

The fault characteristic frequency of ball element is

$$
f_{b}=\frac{D}{2 d}\left(1-\left(\frac{d}{D} \cos \alpha\right)^{2}\right) f_{r}
$$

where $Z$ denotes the number of roller elements, $f_{r}$ is the rotating frequency, $d$ is the roller diameter, $D$ represents the pitch diameter, and $\alpha$ is the contact angle. 


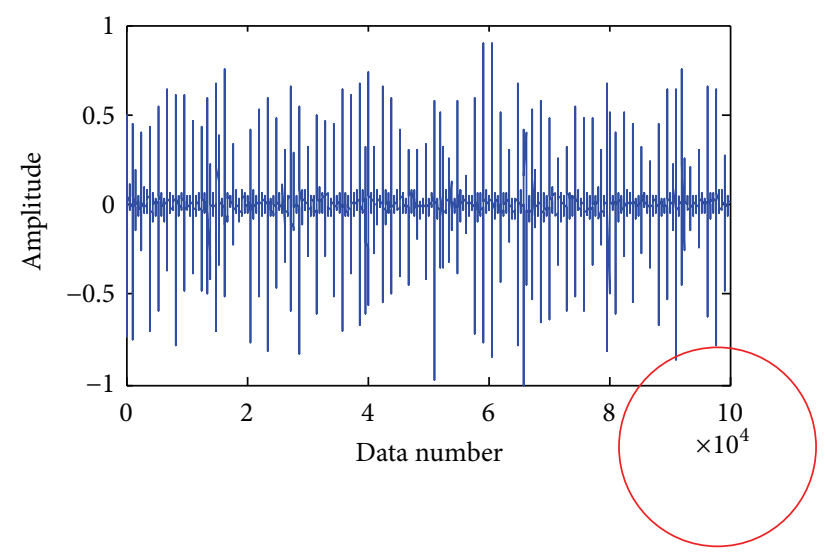

(a)

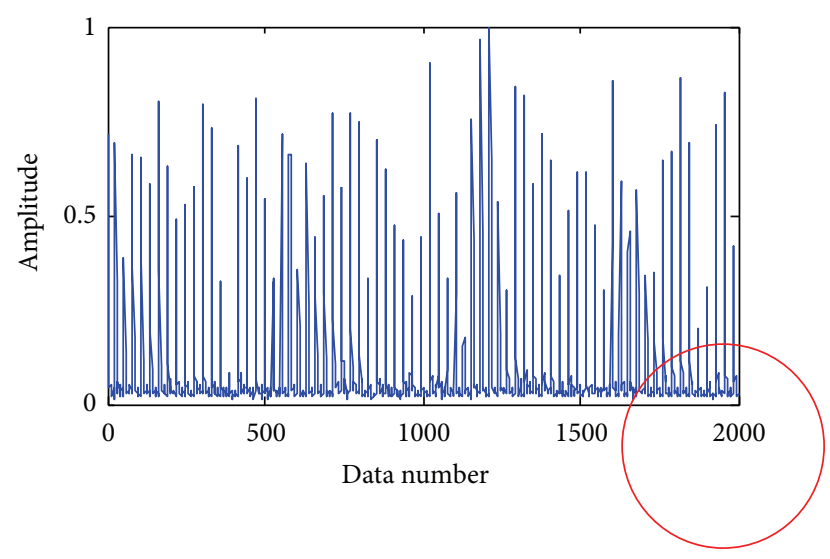

(b)

FIGURE 5: (a) Original signals and (b) symptom parameter wave using peak-to-peak value.

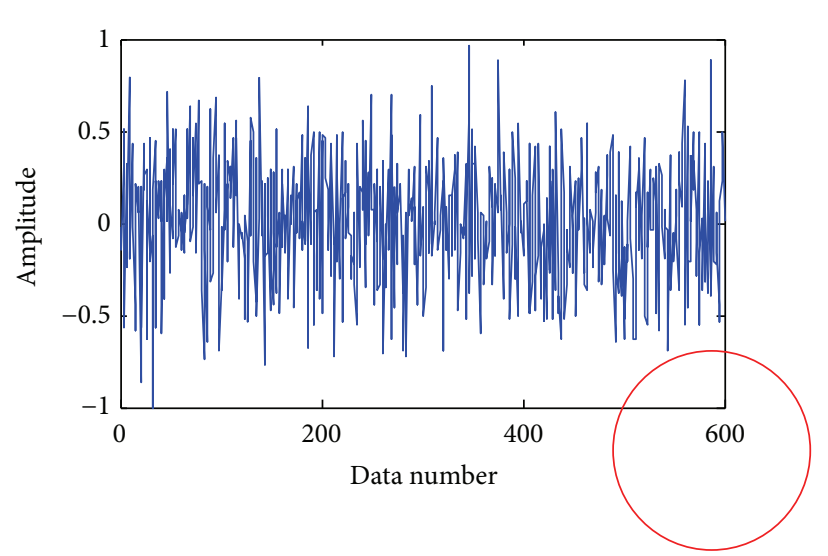

(a)

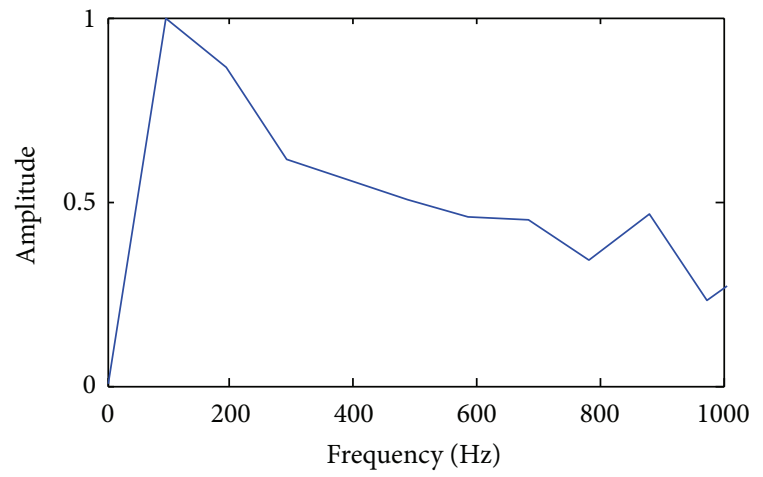

(b)

Figure 6: (a) Random sampling and (b) envelope spectrum of the original signals with 600 points.

4.2. Detection of the Bearing Fault in the Outer Race. The proposed method was employed to detect the bearing fault in the outer race. The peak-to-peak value, kurtosis, and shape factor are considered to represent the original signals, acquiring a symptom parameter wave, aiming at compressing the signals with the significant information preserved. The size of the sliding window is 50 points and the amount of the random sampling points is 600 . All the data in this work are processed through the normalization.

\subsubsection{Detecting the Outer-Race Fault Using Peak-to-Peak Value} and Compressed Sensing. The first time-domain symptom parameter selected to compress the original signals is peakto-peak value, which reflects the impact characteristic of the signals. The time-domain waveform of the original signals is presented in Figure 5(a), which contains plenty of impulses. However, the number of vibration signals sampled for fault diagnosis is 100000 points, which can be compressed to ease the pressure of signal processing. Then the peak-topeak value is utilized to obtain the time-domain symptom parameter wave as shown in Figure 5(b), which retains the impact characteristic of the original signals. Here, the size of the sliding window is 50 points. Thus, the samples are reduced 50 times and the samples are compressed from 100000 to 2000. Next, a Gaussian random matrix is selected as the measurement matrix to reduce the amount of the signals obtained from the envelope of the symptom parameter wave as in Figure 6(a). Envelope analysis is selected as a comparison to validate if it can extract the fault features from the random samples in Figure 6(a). However, it is difficult to find out the frequencies of interest in the envelope analysis of the original signals with only 600 points presented in Figure 6(b). Thus, the detection method based on the CS theory is applied to extract the fault features with the sparsity $K=2$. With the matching pursuit, the fault features are detected successfully, as shown in Figures 7(a) and 7(b), which are almost equal to the theoretical values.

\subsubsection{Detecting the Outer-Race Fault Using Kurtosis and Com-} pressed Sensing. The original signals in this part are presented in Figure 8(a). The kurtosis is used to gain the time-domain symptom parameter wave, assisted by the sliding window 


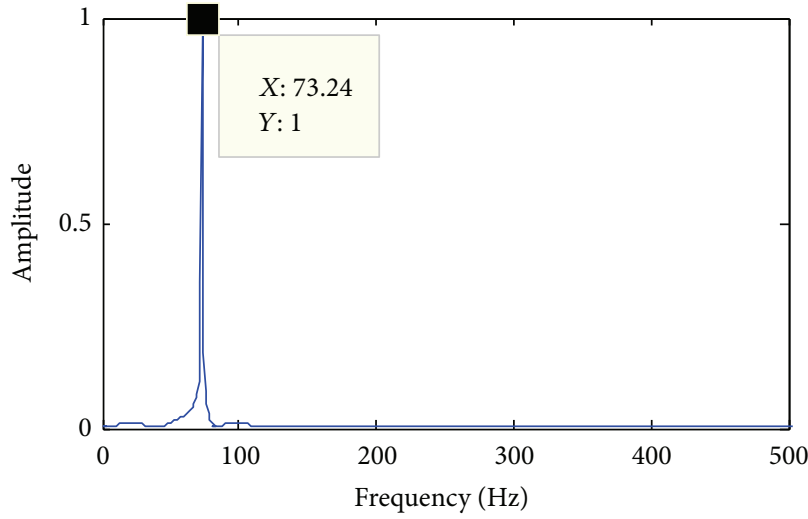

(a)

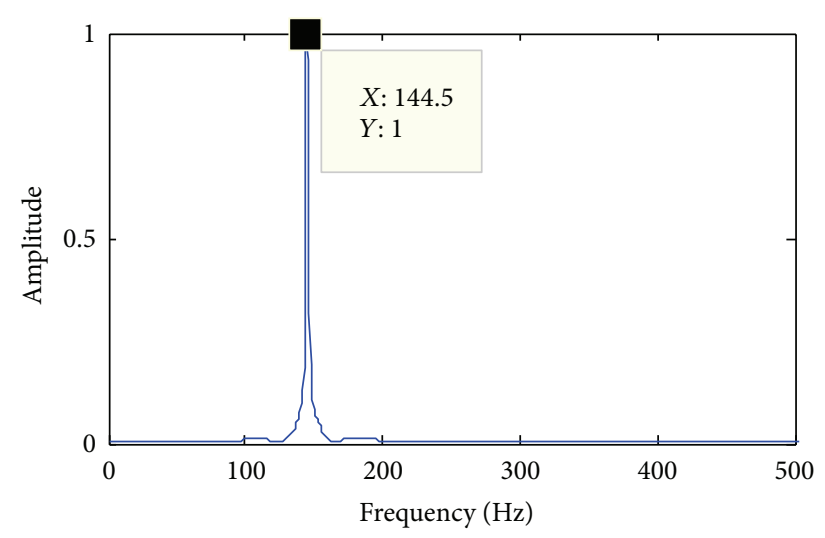

(b)

FIGURE 7: (a) Fault characteristic frequency and (b) twice the fault characteristic frequency.

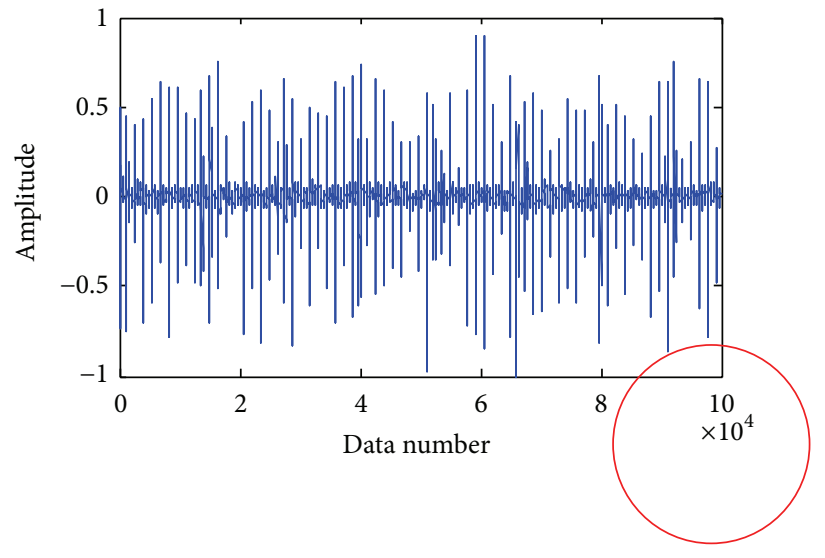

(a)

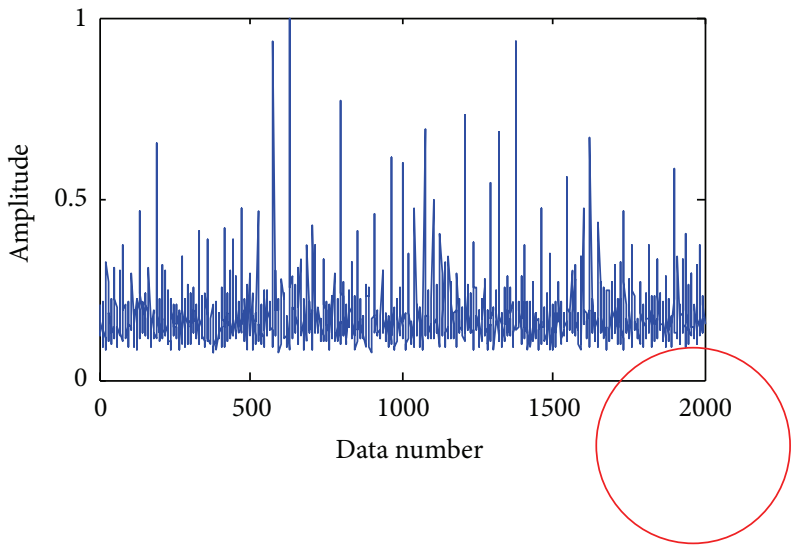

(b)

FIGURE 8: (a) Original signals and (b) symptom parameter wave using kurtosis.

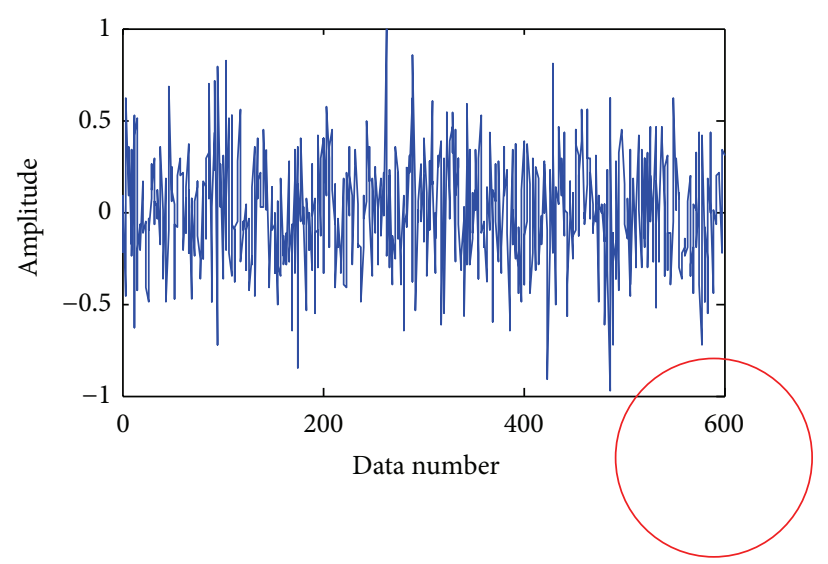

(a)

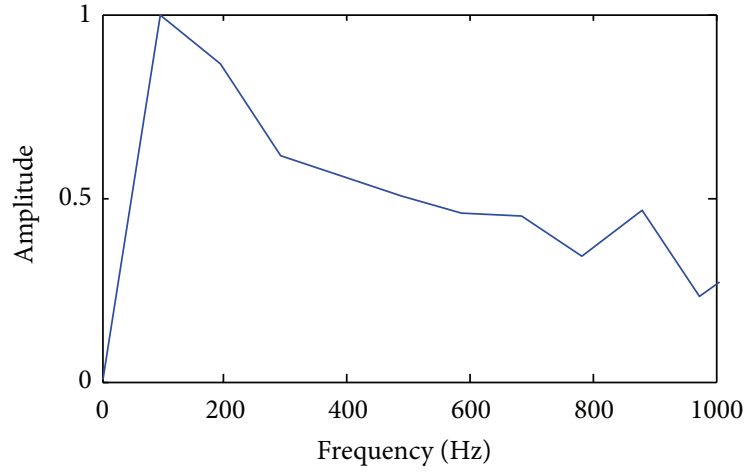

(b)

Figure 9: (a) Random sampling and (b) envelope spectrum of the original signals with 600 points.

with a fixed size of 50 points. The symptom parameter wave is shown in Figure $8(\mathrm{~b})$, which presents that the number of the raw signals is shrunk 50 times. Compared to the symptom parameter wave in Figure 5(b), the impulses in
Figure $8(\mathrm{~b})$ are much less. Then the random sampling was used to further reduce the amount of the symptom parameter wave envelope signals using a measurement matrix and the random sampling waveform is presented in Figure 9(a). The 


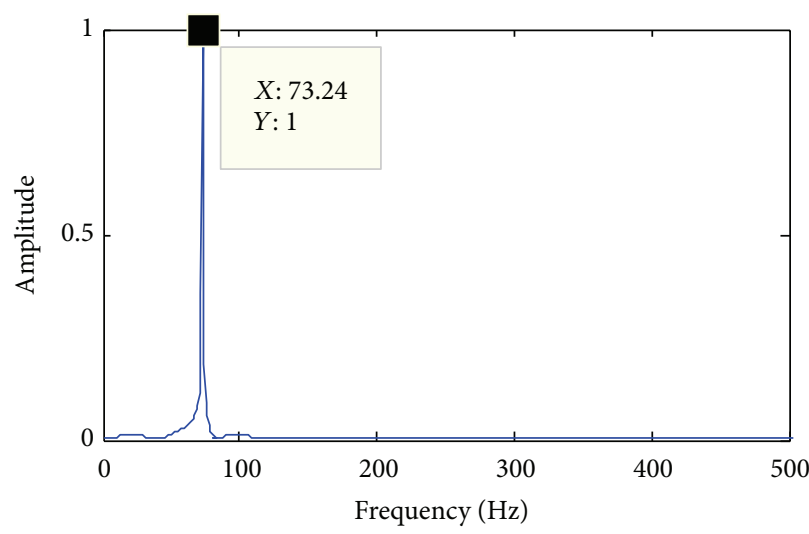

(a)

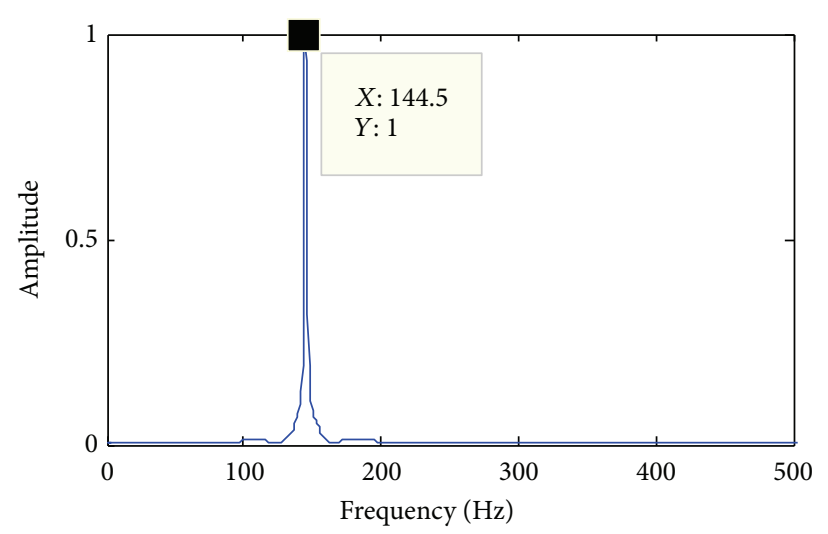

(b)

FIgURE 10: (a) Fault characteristic frequency and (b) twice the fault characteristic frequency.

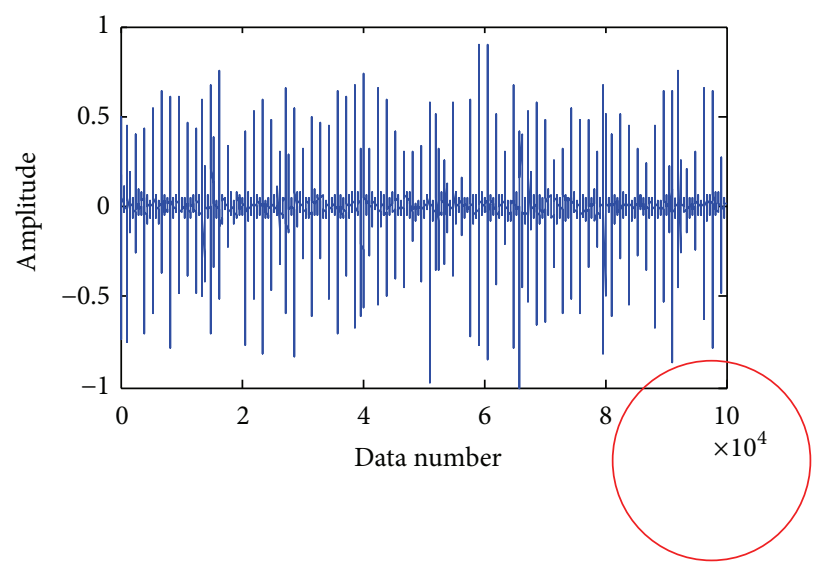

(a)

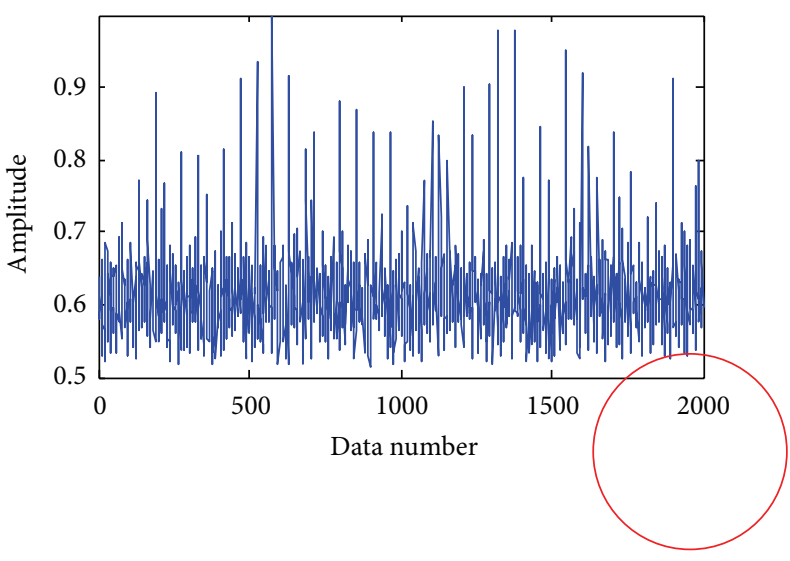

(b)

FIGURE 11: (a) Original signals and (b) symptom parameter wave using shape factor.

envelope analysis of the original signals in Figure 9(a) is presented in Figure 9(b), from which the status of the roller bearing is difficult to identify. However, the fault features are extracted successfully using the proposed method as shown in Figures 10(a) and 10(b), through which the conclusion that there is a fault in outer race can be drawn.

4.2.3. Detecting the Outer-Race Fault Using Shape Factor and Compressed Sensing. The original signals employed here are shown in Figure 11(a). The symptom parameter wave in Figure 11(b) obtained through the shape factor is worse than that in Figure 5(b), and the noise in Figure 11(b) was much more than that in Figure 5(b), since the peak-to-peak value is more sensitive to the impacts. The amount of the target signals is reduced from 100000 to 600 by random sampling, as presented in Figure 12(a). From Figure 12(b), it is difficult to identify the condition of the roller bearing. Finally, the fault features are extracted from the random sampling signals through the proposed method and the fault detection results are shown in Figures 13(a) and 13(b), which illustrate that there is a failure in the outer race.

\section{Comparison between the Three Symptom Parameters}

The amount of the faulty vibration signals is reduced by the time-domain symptom parameters and the CS theory. From the abovementioned results, the three symptom parameters selected to represent the raw vibration signals are all effective in detecting the faults in roller bearings. Here, the detection success ratio is considered as a criterion to compare the effectiveness of the three symptom parameters. The detection success ratio is calculated through 30 groups of vibration signals, containing outer-race fault, inner-race fault, and rolling-element fault. A suitable Gaussian random matrix is selected and it is utilized to detect the remaining samples. When the frequency of interest is detected, this fault detection belongs to a successful example. Different sizes of the sliding window and different dimensions of the measurement matrix are selected to assess the detection success ratio.

5.1. Different Sizes of the Sliding Window. Different sizes of the sliding window with a fixed dimension of the measurement 


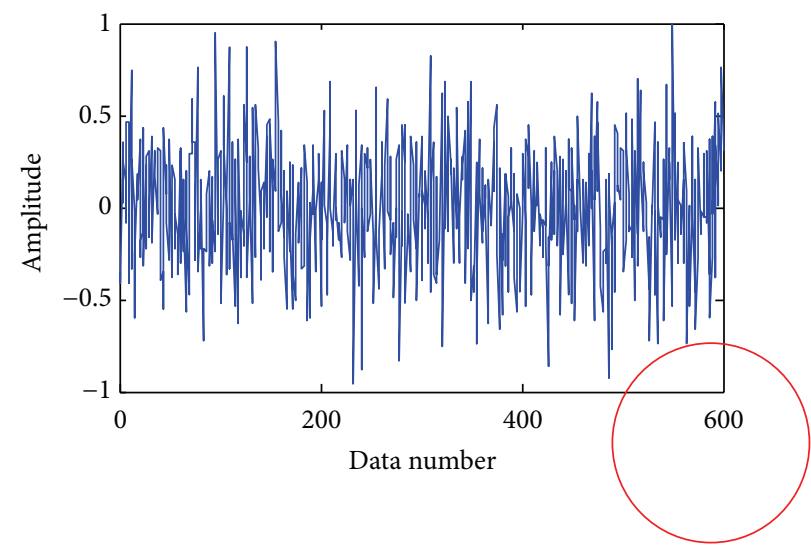

(a)

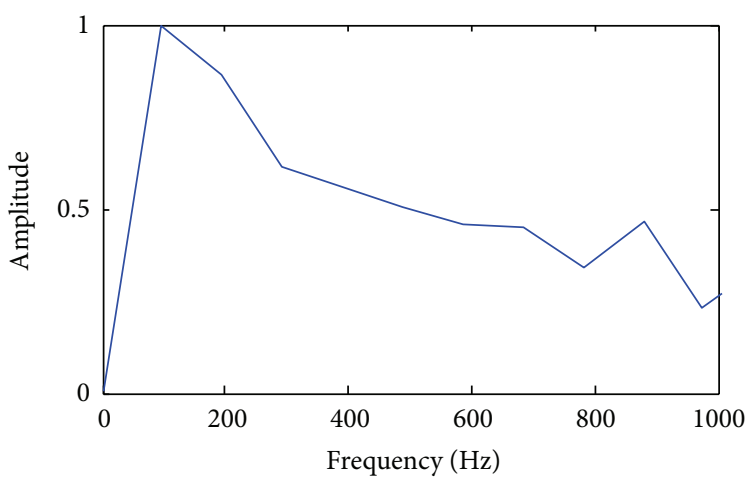

(b)

FIGURE 12: (a) Random sampling and (b) envelope spectrum of the original signals with 600 points.

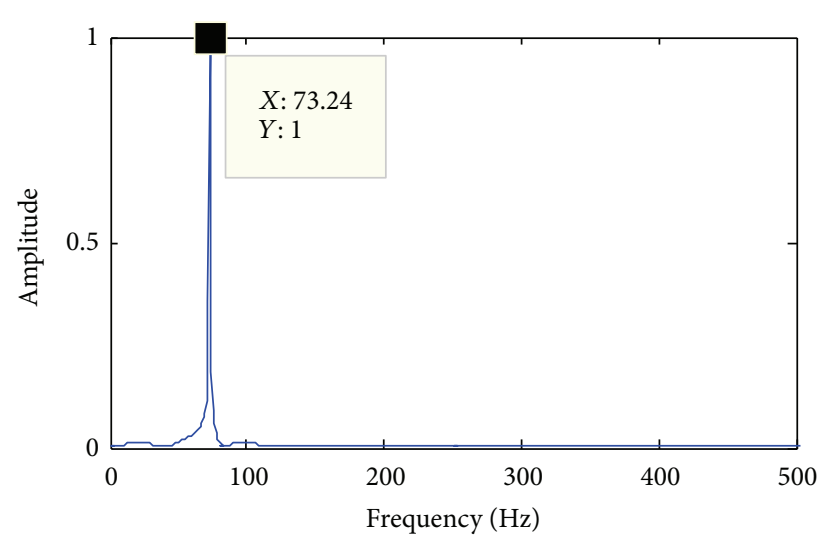

(a)

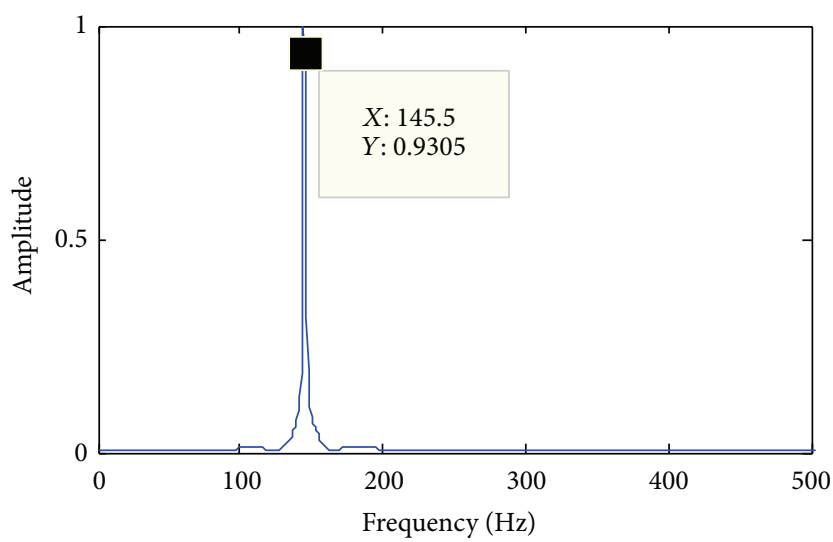

(b)

FIGURE 13: (a) Fault characteristic frequency and (b) twice the fault characteristic frequency.

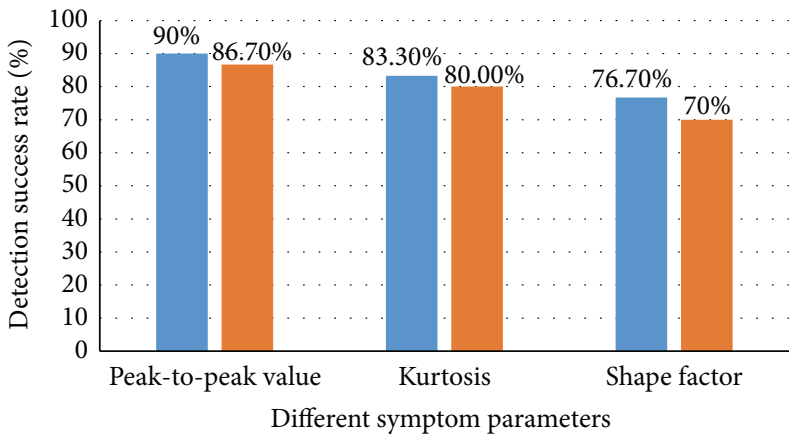

- Size of sliding window: 25 points

- Size of sliding window: 50 points

FIGURE 14: Size of sliding window: 25 points and 50 points.

matrix are utilized to assess the performance of the proposed method. A conclusion can be drawn that the detection success ratio of peak-to-peak value is higher than that of kurtosis and shape factor from Figure 14. Furthermore, the detection success ratio of the sliding window with a 25 -point size is a bit higher than that of the sliding window with a 50-point size. Thus, a conclusion can be drawn that more information related to the roller bearing faults may be collected when a smaller size of the sliding window is selected.

5.2. Different Dimension of the Measurement Matrix. Different dimensions of the measurement matrix with a predetermined size of the sliding window are also employed to evaluate the effectiveness of the proposed fault detection strategy. Also, the detection success ratio of peak-to-peak value is higher than that of kurtosis and shape factor as presented in Figure 15. Moreover, the detection success ratio rises with the increase of the measurement matrix's dimension. This means that when a larger compression ratio is achieved, the detection success ration may decrease. Therefore, if a better compression result needs to be achieved, suitable sizes of the sliding window and measurement matrix should be cooperative.

\section{Conclusion}

In this study, a two-stage compression method is developed to reduce the large amount of the faulty vibration signals 


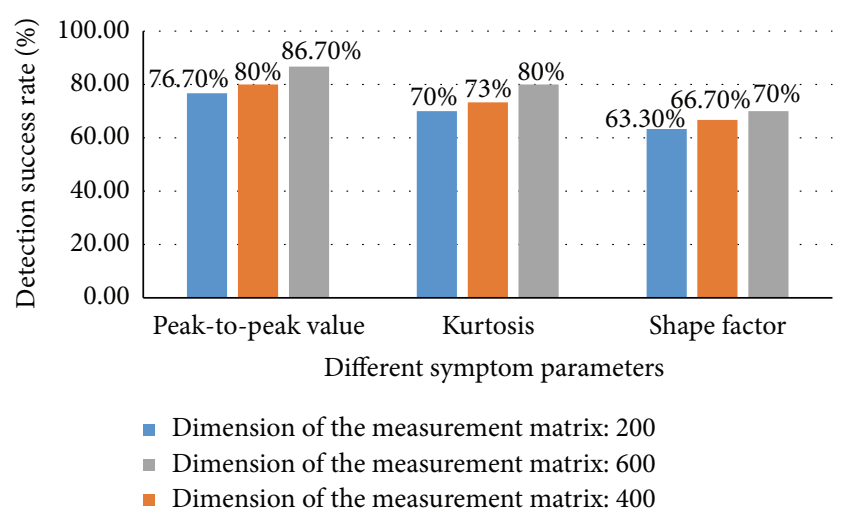

FIGURE 15: Dimension of the measurement matrix: 200, 400, and 600 .

induced by the condition monitoring. First, three timedomain symptom parameters, namely, peak-to-peak value, kurtosis, and shape factor, are separately considered to gain the symptom parameter wave. In this way, the dimension of the original signals is reduced to a certain level but the fault features are reserved. Next, the measurement matrix works as a compressor to further shrink the amount of the symptom parameter wave. Finally, the fault features are extracted via the CS theory from a small number of samples, which were far below the Shannon sampling rate. Compared to the envelope analysis, the proposed method has an advantage in detection faults from limited samples. Detection success ratio is considered as a criterion to compare the three symptom parameters. From the comparing result, a conclusion can be made that the detection success ratio of peak-to-peak value ranked first, followed by that of kurtosis, and the ratio of shape factor was last.

\section{Competing Interests}

The authors declare that there is no conflict of interests regarding the publication of this paper.

\section{Acknowledgments}

This work is partially supported by the National Natural Science Foundation of China (Grant nos. 51405012 and 51375037) and Petro China Innovation Foundation.

\section{References}

[1] Y. B. Li, M. Q. Xu, Y. Wei, and W. H. Huang, "Rotating machine fault diagnosis based on intrinsic characteristic-scale decomposition," Mechanism and Machine Theory, vol. 94, pp. 9-27, 2015.

[2] O. Janssens, R. Schulz, V. Slavkovikj et al., "Thermal image based fault diagnosis for rotating machinery," Infrared Physics and Technology, vol. 73, pp. 78-87, 2015.

[3] H. T. Zhou, J. Chen, G. M. Dong, H. C. Wang, and H. D. Yuan, "Bearing fault recognition method based on neighbourhood component analysis and coupled hidden Markov model,"
Mechanical Systems and Signal Processing, vol. 66-67, pp. 568581, 2016.

[4] A. Mahvash and A. A. Lakis, "Application of cyclic spectral analysis in diagnosis of bearing faults in complex machinery," Tribology Transactions, vol. 58, no. 6, pp. 1151-1158, 2015.

[5] C. Ruiz-Cárcel, V. H. Jaramillo, D. Mba, J. R. Ottewill, and Y. Cao, "Combination of process and vibration data for improved condition monitoring of industrial systems working under variable operating conditions," Mechanical Systems and Signal Processing, vol. 66-67, pp. 699-714, 2016.

[6] N. Saravanan, S. Cholairajan, and K. I. Ramachandran, "Vibration-based fault diagnosis of spur bevel gear box using fuzzy technique," Expert Systems with Applications, vol. 36, no. 2, pp. 3119-3135, 2009.

[7] M. Strączkiewicz, P. Czop, and T. Barszcz, "The use of a fuzzy logic approach for integration of vibration-based diagnostic features of rolling element bearings," Journal of Vibroengineering, vol. 17, no. 4, pp. 1760-1768, 2015.

[8] V. K. Rai and A. R. Mohanty, "Bearing fault diagnosis using FFT of intrinsic mode functions in Hilbert-Huang transform," Mechanical Systems and Signal Processing, vol. 21, no. 6, pp. 2607-2615, 2007.

[9] N. Tandon and A. Choudhury, "Review of vibration and acoustic measurement methods for the detection of defects in rolling element bearings," Tribology International, vol. 32, no. 8, pp. 469-480, 1999.

[10] M. Oltean, J. Picheral, E. Lahalle, H. Hamdan, and J. Griffaton, "Compression methods for mechanical vibration signals: application to the plane engines," Mechanical Systems and Signal Processing, vol. 41, no. 1-2, pp. 313-327, 2013.

[11] V. Sugumaran and K. I. Ramachandran, "Automatic rule learning using decision tree for fuzzy classifier in fault diagnosis of roller bearing," Mechanical Systems and Signal Processing, vol. 21, no. 5, pp. 2237-2247, 2007.

[12] J. H. Zhang, W. P. Ma, J. W. Lin, L. Ma, and X. J. Jia, "Fault diagnosis approach for rotating machinery based on dynamic model and computational intelligence," Measurement, vol. 59, pp. 73-87, 2015.

[13] K. Li, P. Chen, S. Wang, and H. Wang, "Intelligent diagnosis method for bearing using non-dimensional symptom parameters and Ant colony optimization. International Information Institute (Tokyo)," Information, vol. 15, no. 2, pp. 867-877, 2012.

[14] Y. J. Wang, Y. C. Jiang, and S. Q. Kang, "The applications of time domain and frequency domain statistical factors on rolling bearing performance degradation assessment," Computer Modeling and New Technologies, vol. 18, no. 8, pp. 192-198, 2014.

[15] X. W. Niu, L. M. Zhu, and H. Ding, "New statistical moments for the detection of defects in rolling element bearings," The International Journal of Advanced Manufacturing Technology, vol. 26, no. 11-12, pp. 1268-1274, 2005.

[16] R. B. W. Heng and M. J. M. Nor, "Statistical analysis of sound and vibration signals for monitoring rolling element bearing condition," Applied Acoustics, vol. 53, no. 1-3, pp. 211-226, 1998.

[17] Y. Guo, J. Na, B. Li, and R.-F. Fung, "Envelope extraction based dimension reduction for independent component analysis in fault diagnosis of rolling element bearing," Journal of Sound and Vibration, vol. 333, no. 13, pp. 2983-2994, 2014.

[18] H.-Q. Wang, W. Hou, G. Tang, H.-F. Yuan, Q.-L. Zhao, and $\mathrm{X}$. Cao, "Fault detection enhancement in rolling element bearings via peak-based multiscale decomposition and envelope demodulation," Mathematical Problems in Engineering, vol. 2014, Article ID 329458, 11 pages, 2014. 
[19] H. Q. Wang and P. Chen, "A feature extraction method based on information theory for fault diagnosis of reciprocating machinery," Sensors, vol. 9, no. 4, pp. 2415-2436, 2009.

[20] Q. Gao, C. Duan, H. Fan, and Q. Meng, "Rotating machine fault diagnosis using empirical mode decomposition," Mechanical Systems and Signal Processing, vol. 22, no. 5, pp. 1072-1081, 2008.

[21] Z. K. Peng, P. W. Tse, and F. L. Chu, "A comparison study of improved Hilbert-Huang transform and wavelet transform: application to fault diagnosis for rolling bearing," Mechanical Systems and Signal Processing, vol. 19, no. 5, pp. 974-988, 2005.

[22] J. Ma, J. Wu, and X. Yuan, "The fault diagnosis of the rolling bearing based on the LMD and time-frequency analysis," International Journal of Control and Automation, vol. 6, no. 4, pp. 357-376, 2013.

[23] M. J. Li, H. Q. Wang, G. Tang, H. F. Yuan, and Y. Yang, "An improved method based on CEEMD for fault diagnosis of rolling bearing," Advances in Mechanical Engineering, vol. 2014, Article ID 676205, 10 pages, 2014.

[24] J.-H. Ahn, D.-H. Kwak, and B.-H. Koh, "Fault detection of a roller-bearing system through the EMD of a wavelet denoised signal," Sensors, vol. 14, no. 8, pp. 15022-15038, 2014.

[25] D. L. Donoho, "Compressed sensing," IEEE Transactions on Information Theory, vol. 52, no. 4, pp. 1289-1306, 2006.

[26] A. S. Khwaja and J. Ma, "Applications of compressed sensing for sar moving-target velocity estimation and image compression," IEEE Transactions on Instrumentation and Measurement, vol. 60, no. 8, pp. 2848-2860, 2011.

[27] S. Zhu, B. Zeng, and M. Gabbouj, "Adaptive sampling for compressed sensing based image compression," Journal of Visual Communication and Image Representation, vol. 30, pp. 94-105, 2015.

[28] I. Kim and N.-L. Vu, "parallel compressed sensing method to accelerate MRI," in Proceedings of the 12th International Conference on Control, Automation and Systems (ICCAS '12), pp. 1103-1107, JeJu Island, Republic of Korea, October 2012.

[29] M. Ghahremani and H. Ghassemian, "Remote sensing image fusion using ripplet transform and compressed sensing," IEEE Geoscience and Remote Sensing Letters, vol. 12, no. 3, pp. 502506, 2015.

[30] K. P. Zhu, X. Lin, K. X. Li, and L. L. Jiang, “Compressive sensing and sparse decomposition in precision machining process monitoring: from theory to applications," Mechatronics, vol. 31, pp. 3-15, 2015.

[31] X. Chen, Z. Du, J. Li, X. Li, and H. Zhang, "Compressed sensing based on dictionary learning for extracting impulse components," Signal Processing, vol. 96, pp. 94-109, 2014.

[32] G. Tang, Q. Yang, H.-Q. Wang, G.-G. Luo, and J.-W. Ma, "Sparse classification of rotating machinery faults based on compressive sensing strategy," Mechatronics, vol. 31, pp. 60-67, 2015.

[33] Y. Wang, J. Xiang, Q. Mo, and S. He, "Compressed sparse timefrequency feature representation via compressive sensing and its applications in fault diagnosis," Measurement, vol. 68, pp. 7081, 2015.

[34] L. Stankovic, S. Stankovic, and M. Amin, "Missing samples analysis in signals for applications to L-estimation and compressive sensing," Signal Processing, vol. 94, no. 1, pp. 401-408, 2014.

[35] S. Gishkori, G. Leus, and V. Lottici, "Compressive sampling based differential detection for UWB impulse radio signals," Physical Communication, vol. 5, no. 2, pp. 185-195, 2012.

[36] G. Tang, W. Hou, H. Q. Wang, G. G. Luo, and J. W. Ma, "Compressive sensing of roller bearing faults via harmonic detection from under-sampled vibration signals," Sensors, vol. 15, no. 10, pp. 25648-25662, 2015.

[37] H. Q. Wang, R. T. Li, G. Tang, H. F. Yuan, Q. L. Zhao, and X. Cao, "A Compound fault diagnosis for rolling bearings method based on blind source separation and ensemble empirical mode decomposition," PLoS ONE, vol. 9, no. 10, Article ID e109166, 2014. 


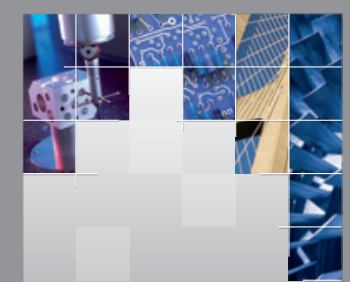

\section{Enfincering}
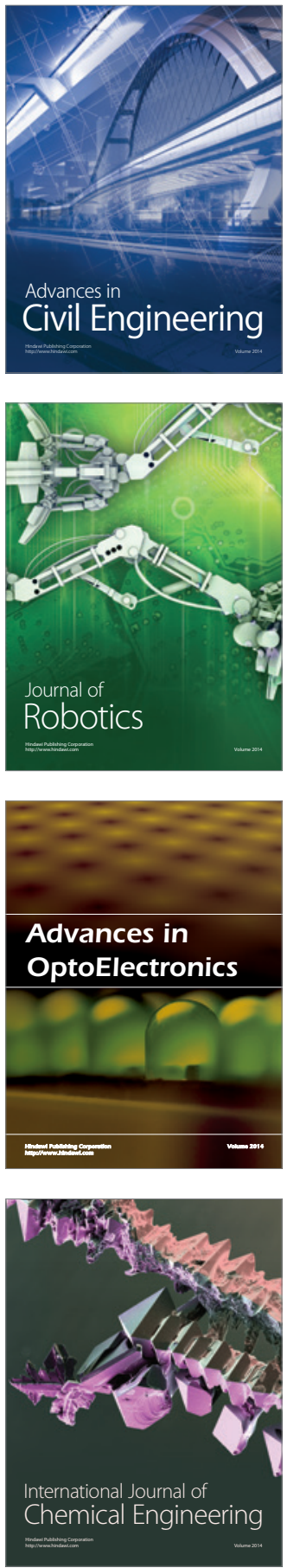

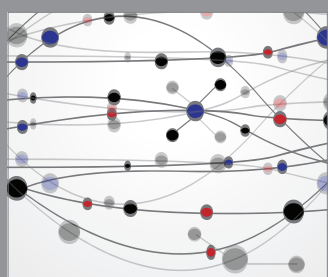

The Scientific World Journal

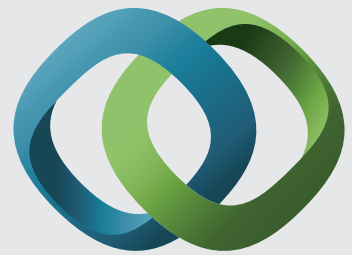

\section{Hindawi}

Submit your manuscripts at

http://www.hindawi.com
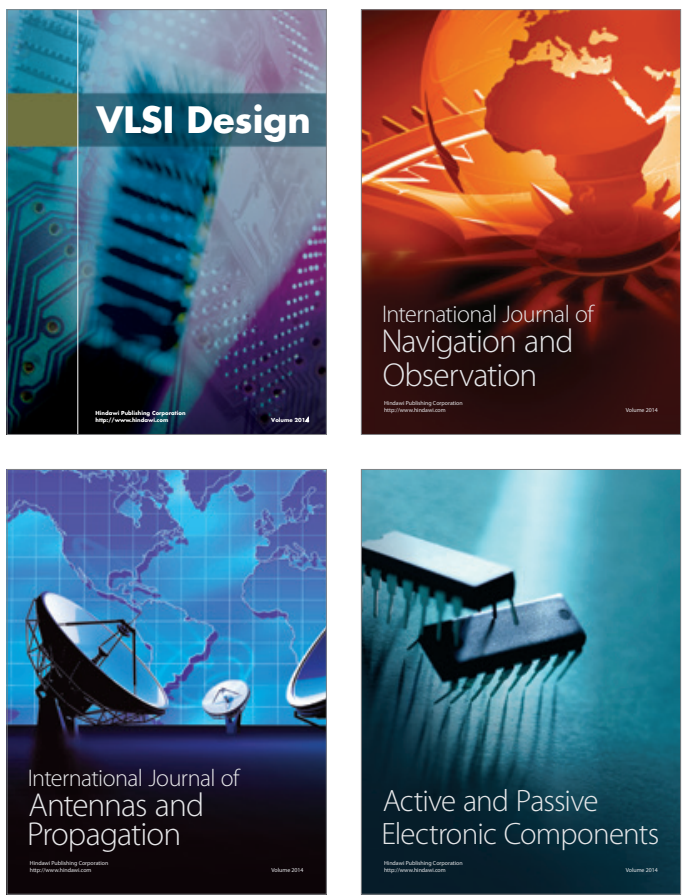
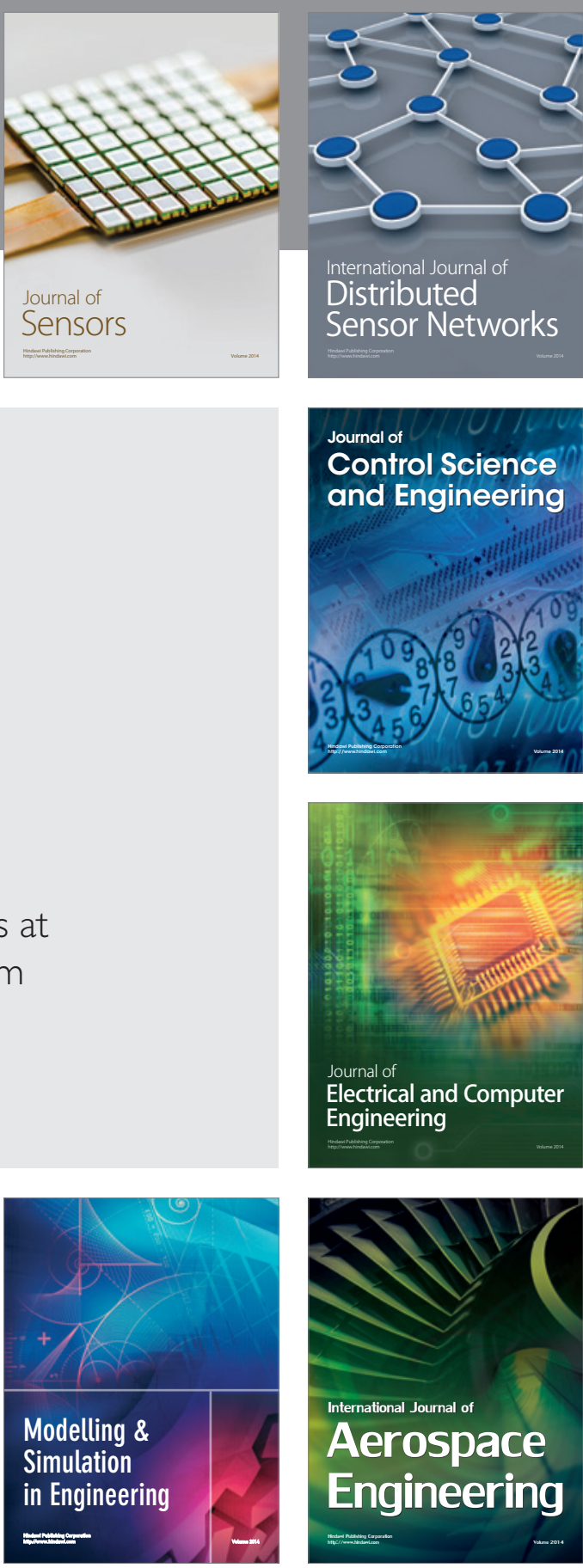

International Journal of

Distributed

Sensor Networks

Journal of

Control Science

and Engineering
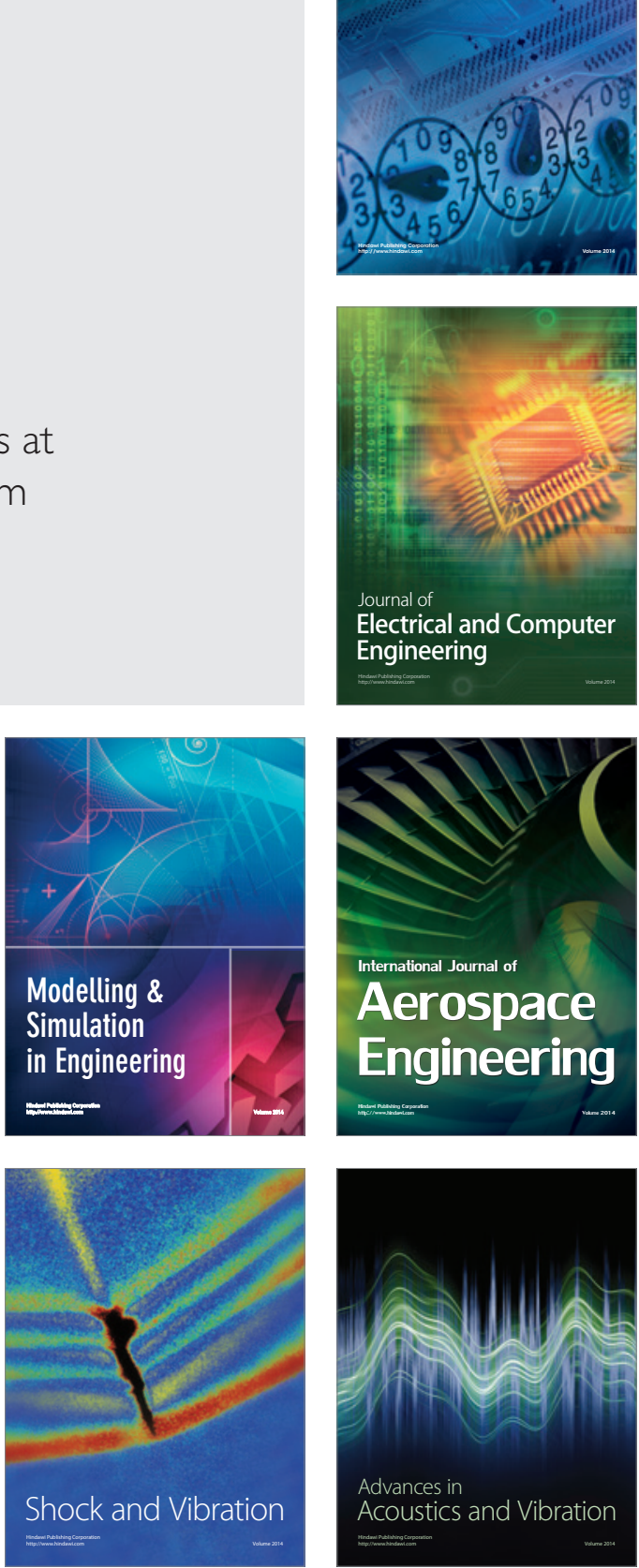